\title{
Qualität messen oder Qualität bewerten
}

Christoph Rehmann-Sutter

Korrespondenz:

Prof. Dr. phil.

Christoph Rehmann-Sutter

Universität Basel

Arbeitsstelle für Ethik

in den Biowissenschaften

Schönbeinstrasse 20

CH-4056 Basel

christoph.rehmann-sutter@unibas.ch
Alle wollen Qualität. Wenn schon, dann etwas Gutes! Ob es sich um Gemüse zum Kochen, neue Fenster fürs Haus, eine ärztliche Behandlung, Spitalpflege, einen Krimi oder nur um eine Flasche Rotwein handelt, immer wollten wir Qualität. Die Kriterien unterscheiden sich freilich beträchtlich, je nachdem, worum es sich handelt. Die Wahl der Kriterien und ihre genaue Ausgestaltung sind eine sensible Sache und haben auch einiges mit Ethik zu tun: Ethik der Gemüseproduktion, Ethik der Medizin, Ethik der Pflege, Ethik des Weinbaus usw. Immer sind Überlegungen darüber nötig, was diejenigen, die ein Produkt nutzen, brauchen. Damit ist mit Qualität eigentlich eine ethische Beziehung der Verantwortlichkeit gemeint.

Die aktuellen Wünsche der Kundinnen, Konsumenten, Leserinnen, Patienten zählen. Das sind ihre Präferenzen, ihr Kaufverhalten, ihre Bestellungen, ihre Aufträge, ihre Wahl. Aber immer sind auch weitergehende Überlegungen wichtig, die auf die «besten Interessen» der jeweiligen Nutzer zielen. Nicht immer kommen die besten Interessen mit den aktuellen Wünschen zur Deckung. Man kann davon ausgehen, dass Menschen einen günstigen Preis bevorzugen, aber das ist nicht alles: Sie wollen auch gesund essen und trinken, sie wollen einen köstlichen Geschmack, sie wollen von einer gehaltvollen Geschichte überrascht, bereichert und nicht am Schluss enttäuscht werden. Ähnliches gilt auch für die Medizin. Qualität bezieht sich auf die besten Interessen.

Interessant ist übrigens, welche Worte man für die Nutzer in den verschiedenen Fällen verwendet, denn das zeigt schon etwas davon auf, in welchen Kategorien man ihre besten Interessen einordnet. Patientinnen und Patienten sind etwas anderes als Kunden oder Konsumentinnen.

In der Medizin sind Qualitätsüberlegungen besonders anspruchsvoll, vielschichtig und ethisch gesehen besonders spannend. Was ist z. B. wichtiger: schnell gesund werden oder länger gesund bleiben? Je nachdem, was wichtiger ist, können sich Behandlungspläne unterscheiden. Das ist das Dilemma der Effizienz. Es gibt auch ein Dilemma der Lebensqualität. Was ist wichtiger: weiterzuleben oder die Bedingungen, unter denen das Leben weitergehen kann? Wir wollen zwar weiterleben, aber nicht zu allen Bedingungen. Die Lebensqualität ist wichtig und sie steht

\section{Mesurer ou évaluer la qualité}

II n'est pas possible de mesurer la qualité au sens propre du terme, mais seulement de l'évaluer. On mesure la qualité d'un produit ou d'une prestation à la façon dont ils répondent aux souhaits de leurs utilisateurs, mais aussi à la manière dont ils servent leurs intérêts. Des exigences élevées prenant en compte les aspects éthiques président aux stratégies de mesure, assurance et gestion de la qualité. Evaluer la qualité dans le domaine de la santé ne va pas sans embûches. L'efficience, la qualité de vie et les mesures elles-mêmes posent autant de dilemmes. Tout mesurage présuppose une évaluation: quels sont les paramètres qui comptent et comment doivent-ils compter?

quer zur Lebensverlängerung. Man kann sie nicht so einfach miteinander aufwiegen, wenn man das überhaupt kann.

\section{Das Dilemma der Messung}

Qualität messbar machen, geht das überhaupt? Messen bedeutet ja nichts anderes als eine quantitative Bewertung. Einer Qualität wird eine Quantität zugeordnet, eine Zahl oder ein Cluster von Zahlen. Das Eigentliche der Qualität ist aber genau das, was sich nicht quantitativ ausdrücken lässt.

Oft kommt das Bedürfnis der quantitativen Bewertung von Qualität aus einem Bedürfnis nach Vergleichbarkeit. Aber das ist tückisch. Stellen wir uns vor, jemand würde herausfinden wollen, welches die besten Violinsonaten des Barock sind und er würde einen zusammengesetzten Qualitätsindex erstellen, in dem die Innovativität der kompositorischen Ideen, die Häufigkeit, mit der Motive repetiert werden, die Unkonventionalität der Harmoniewechsel und ähnliche Faktoren vorkommen. Tatsächlich würden vielleicht die Violinsonaten von Johann Sebastian Bach in einem Score bei den Besten abschneiden. Alle wären dann zufrieden, weil dann nämlich bestätigt wäre, was man vorher 
schon wusste. Man wusste es, aber aus anderer Quelle sozusagen, nämlich aus der Quelle eines umfassenden Qualitätssensoriums für Musik, in das ganz viele unquantifizierbare, intuitive und emotionale Elemente des Musikerlebnisses hineinspielen. Was wäre nämlich, wenn Bach nicht ganz oben abschneiden würde? Woran würde man dann eher zweifeln? Am eigenen musikalischen Erlebnis oder am Bewertungsverfahren? Ich vermute (und hoffe es gleichzeitig inständig), man würde dann an der Zuverlässigkeit der Messanordnung zweifeln.

Auch im Gesundheitswesen wird viel verglichen, gemessen und quantitativ bewertet. Die Tendenz ist steigend. Im Zuge der Einführung der DRG als Bemessungsinstrument ist eine noch stärkere Quantifizierung von Behandlungsqualität unumgänglich. Nicht zum Vergnügen, sondern letztlich aus Verzweiflung, nämlich aus finanziellem Druck. Es kann sein, dass es ähnlich $\mathrm{zu}$ und her gehen wird, wie mit Bachs Violinsonaten.

Man kann natürlich eine Menge anderer Beispiele herbeiziehen, um zu untersuchen, wie sich das Quantifizierbare aus der Qualität ergibt oder wie sich die Qualität hinter der Quantifizierbarkeit gerade verbirgt. Nehmen wir die Schulnoten. Noten geben ist tückisch, wenn auch nicht unmöglich. Aber es ist doch wichtig, in Erinnerung zu behalten, dass Noten tückisch sind und täuschen können. Manch einer ist gerade in einem Fach später gut geworden, in dem er schlechte Noten hatte. Und manch eine ist in einem musischen Fach ungerecht bewertet worden, nur weil man auf gewisse äusserliche Merkmale achtete, die sich zählen und objektivieren lassen. Oder ein Beispiel, das den Akademikerinnen und Akademikern heute wohlvertraut ist: Die Bewertung der Qualität von Forschung nach den impact factors der Journale, in denen Resultate publiziert werden und nach der Anzahl der Publikationen. Darüber wird im Moment viel geschrieben und ich enthalte mich eines abschliessenden Urteils. Aber die Vermutung werde ich nicht los, dass man nämlich eine Reihe der historisch bedeutenden wissenschaftlichen Innovationen mit diesen Methoden gar nicht erfasst oder

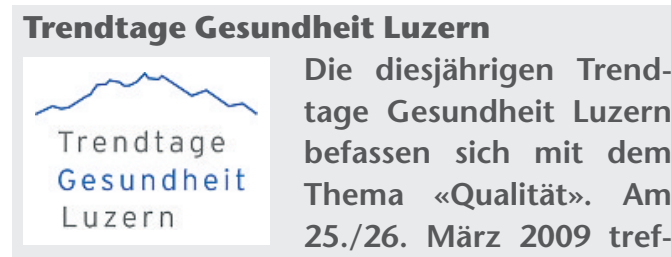

fen sich im KKL Luzern Fachleute aus Medizin und Gesundheitswesen, Vertreter aus Politik, schlecht eingestuft hätte. Man denke an Mönch Gregor Mendels Arbeiten, die zuerst über Jahre unbeachtet blieben, dann aber eine Revolution der Biologie und Medizin ausgelöst haben. Wie wäre Kant bewertet worden? Bücher gehen kaum in die Bewertungsverfahren ein. Wie könnte man aber gute Philosophie entwickeln, ohne Bücher zu schreiben?

Der Teufel der Qualitätsmessung steckt nicht im Versuch der Bewertung und im Interesse an Transparenz. Der Teufel steckt vielmehr im Vergessen dessen, wie man bewertet, wenn man so oder so misst. Bewerten kommt hier im eigentlichen Sinn des Wortes vor. Das Anlegen eines Massstabes ist eine Bewertung, eine Qualifizierung: nämlich die, dass die Länge, die man mit dem Massstab misst, ein relevantes, d.h. qualitätskonstitutives Merkmal ist. Das eigentliche Bewerten in der Messung geschieht nicht beim Ablesen des Instruments, also beim Zuordnen eines Wertes (einer Messzahl) zum Gemessenen. Das eigentliche Bewerten findet vorher statt, nämlich bei der Konstruktion der Messanordnung. Das Nachdenken über die Tücken der Qualitätsmessungen führt deshalb nicht $\mathrm{zu}$ einer Ablehnung der Bewertungsverfahren, sondern zu einer Forderung nach Transparenz hinsichtlich der Werte, die in die Messungen einfliessen. Messen setzt Bewertung voraus. Man setzt voraus, dass bestimmte messbare Charakteristika der zu bewertenden Sache wertvoll sind oder sie in ihrem Wert auszeichnen.

Diese Überlegung wirft auch ein Licht auf die Anliegen des Qualitätsmanagements und der Qualitätssicherung im Gesundheitswesen. Qualitätssicherung ist eine sehr anspruchsvolle Aufgabe, möchte ich sagen, weil sie ein Bewusstsein der zunächst meistens verborgenen Bewertungen verlangt, ja voraussetzt, die im Messen von Qualität drinsteckt. Qualitätsmanagement verlangt eine Wahrnehmung der Bedürfnisse der Patientinnen und Patienten. Diese Bedürfnisse spiegeln sich nicht in ihrem Wahlverhalten (als Konsumenten von Gesundheitsleistungen), sondern betreffen auch ihre «besten Interessen». Deshalb ist Qualitätsmanagement eine ethisch anspruchsvolle Herausforderung.

Verwaltung und Ökonomie sowie wissenschaftliche Kreise zu einem öffentlichen Symposium, um über Qualität im Spannungsfeld von «Machbarkeit, Finanzierbarkeit und Ethik» zu diskutieren. Bereits haben sich mehr als 200 Personen angemeldet. Informationen und Anmeldung unter www.trendtage-gesundheit.ch. 